\title{
Chromosome Level Genome Assembly and Comparative Genomics between Three Falcon Species Reveals an Unusual Pattern of Genome Organisation
}

\author{
Sunitha Joseph ${ }^{1,2}$, Rebecca E. O'Connor ${ }^{1}$, Abdullah F. Al Mutery ${ }^{1,2}$, Mick Watson ${ }^{3}$, \\ Denis M. Larkin ${ }^{4}$ and Darren K. Griffin ${ }^{1, *}$ \\ 1 School of Biosciences, University of Kent, Canterbury CT2 7NJ, UK; sjoseph@cvrl.ae (S.J.); \\ R.O'Connor@kent.ac.uk (R.E.O.); abdullah.almutery@gmail.com (A.F.A.M.) \\ 2 Central Veterinary Research Laboratories and Dubai Equine Hospital, University of Sharjah, Sharjah, UAE \\ 3 Department of Genomics and Genetics, The Roslin Institute and Royal (Dick) School of Veterinary Studies, \\ University of Edinburgh, Midlothian EH25 9RG, UK; mick.watson@roslin.ed.ac.uk \\ 4 Department of Comparative Biomedical Sciences, Royal Veterinary College, University of London, \\ London NW1 0TU, UK; dlarkin@rvc.ac.uk \\ * Correspondence: d.k.griffin@kent.ac.uk
}

Received: 29 August 2018; Accepted: 29 September 2018; Published: 18 October 2018

\begin{abstract}
Whole genome assemblies are crucial for understanding a wide range of aspects of falcon biology, including morphology, ecology, and physiology, and are thus essential for their care and conservation. A key aspect of the genome of any species is its karyotype, which can then be linked to the whole genome sequence to generate a so-called chromosome-level assembly. Chromosome-level assemblies are essential for marker assisted selection and genotype-phenotype correlations in breeding regimes, as well as determining patterns of gross genomic evolution. To date, only two falcon species have been sequenced and neither initially were assembled to the chromosome level. Falcons have atypical avian karyotypes with fewer chromosomes than other birds, presumably brought about by wholesale fusion. To date, however, published chromosome preparations are of poor quality, few chromosomes have been distinguished and standard ideograms have not been made. The purposes of this study were to generate analyzable karyotypes and ideograms of peregrine, saker, and gyr falcons, report on our recent generation of chromosome level sequence assemblies of peregrine and saker falcons, and for the first time, sequence the gyr falcon genome. Finally, we aimed to generate comparative genomic data between all three species and the reference chicken genome. Results revealed a diploid number of $2 n=50$ for peregrine falcon and $2 n=52$ for saker and gyr through high quality banded chromosomes. Standard ideograms that are generated here helped to map predicted chromosomal fragments (PCFs) from the genome sequences directly to chromosomes and thus generate chromosome level sequence assemblies for peregrine and saker falcons. Whole genome sequencing was successful in gyr falcon, but read depth and coverage was not sufficient to generate a chromosome level assembly. Nonetheless, comparative genomics revealed no differences in genome organization between gyr and saker falcons. When compared to peregrine falcon, saker/gyr differed by one interchromosomal and seven intrachromosomal rearrangements (a fusion plus seven inversions), whereas peregrine and saker/gyr differ from the reference chicken genome by $14 / 13$ fusions (11 microchromosomal) and six fissions. The chromosomal differences between the species could potentially provide the basis of a screening test for hybrid animals.
\end{abstract}

Keywords: falcons; genome; chromosomes; karyotype; avian 


\section{Introduction}

Study of the genomics of falcon species is important for understanding a wide range of aspects of falcon biology, including morphology, ecology, and physiology, as well as being essential for conservation efforts. Whole genome sequencing enables us to understand how the genome relates to phenotype, e.g., to growth, development, maintenance, and disease resistance [1]. Understanding the genome also helps us to study the regulatory regions and "nonsense" regions, comparing genes across species and identifying genetic variants that lead to certain traits [2]. In addition to the sequence itself, a key aspect of the genomics of any species is its karyotype [3].

The karyotype is essentially the organization of the genome expressed as an arrangement of chromosomes (usually smallest to largest). The ultimate aim of any de novo genome sequencing effort therefore is to assign all or most of the sequences to the appropriate chromosomes in the karyotype, with each gene or marker in order-in essence, creating a genomic map [4]. Making a map of the genome in relation to the karyotype (a so-called chromosome-level assembly) can be useful for genotype-phenotype correlations, followed by marker assisted selection in breeding regimes [5]. Similarly, with chromosome-level assemblies, we can determine patterns of gross genomic evolution between species [1]. Despite this, many animals, although sequenced, do not have a chromosome-level genome assembly [6], and until recently, this also applied to falcons. The purposes of this study are therefore both to report on our very recent results in creating chromosome-level assemblies for two falcon species, as well as presenting hitherto unpublished results on chromosome description and comparative genomics between three of the best-known species.

Avian genomes are usually characterized by the large variation in chromosome size, with most species having $\sim 10$ pairs of macrochromosomes and $\sim 30$ pairs of microchromosomes. The majority of avian karyotypes typically have large number of chromosomes, more than most other vertebrates, with a diploid count of $\sim 2 n=80$ in two-thirds of species [7]. The Falco genus is however unusual in having lower diploid chromosome numbers ranging from 40-54, with 7-11 pairs of large and medium-sized chromosomes, and about 13-16 pairs of microchromosomes [8]. Karyotypes of peregrine falcon and prairie falcons purportedly are similar, and, according to Schmutz and Oliphant [9], differ from gyr falcons by four chromosomes. However, the karyotypes currently available in the literature are of poor quality. Amaral and Jorge summarized karyotypes of 66 species of Falconiformes that were analyzed between 1966 to 2001 and their results revealed that the low diploid numbers in Falco species suggest extensive translocation, as well as the fusion of the microchromosomes into larger chromosomes [10]. Depending on classification, the Falco genus comprises 37 or 39 species [11-13], from which 10 species have reported karyotype data. They are Falco columbarius (merlin) $(2 n=40)$, Falco mexicanus (prairie falcon) $(2 n=48)$, Falco chicquera (red-necked falcon) $(2 n=50)$, Falco jugger (laggar falcon) $(2 n=50)$, Falco sparverius (American kestrel) $(2 \mathrm{n}=50)$, Falco subbuteo (Eurasian hobby) $(2 \mathrm{n}=50)$, Falco peregrinus (peregrine falcon) $(2 \mathrm{n}=50)$, Falco rusticolus (gyr falcon) $(2 \mathrm{n}=52)$, Falco tinunculusi (Common kestrel) $(2 \mathrm{n}=52)$, and Falco biarmicus (lanner falcon) $(2 \mathrm{n}=52$ or 54$)$ [9,14-17].

Among falcon species the most comprehensive account to date of the relationship between their chromosomes and those of other birds is given by Nishida et al. [17]. In that study, molecular cytogenetic characterization of the chromosomal homologies of three Falco species, the common kestrel (F. tinnunculus), Peregrine falcon (F. peregrinus), and merlin falcon (F. columbarius) characterization was performed while using chromosome paints derived from chicken chromosomes 1-9 and Z. F. tinnunculus has a karyotype $(2 \mathrm{n}=52)$ consisting of all acrocentric (one arm, centromere at the top) chromosomes, except for the submetacentric (bi-armed) W chromosome. F. peregrinus has a diploid number of $(2 n=50)$, all acrocentric chromosomes except for the one pair of large sub-metacentric macrochromosomes. F. columbarius has a lower chromosome number $(2 \mathrm{n}=40)$, and, unlike those of other species, has six pairs of large bi-armed (sub-metacentric) chromosomes. Nishida et al. [17] therefore suggested that the ancestral karyotype of Falco probably had a diploid number of $2 n=52$ or 54, consisting of all acrocentric chromosomes, except for the $\mathrm{W}$ chromosome. F. tinnunculus is considered to have retained the most of the ancestral status of Falconidae karyotypes [17]. Until recently, 
however, comparative studies have been limited to the largest chromosomes $(1-9+Z)$ using whole chromosome paints.

Peregrine (F. peregrinus) and saker (F. cherrug) genomes were sequenced around five years ago in an attempt to understand evolutionary aspects of predatory adaptations of falcons [18]. Sequencing of males of both species was achieved by a next-generation genome sequencing platform, generating genome sizes of both species estimated at $1.2 \mathrm{~Gb}$ with a genome coverage of $106.72 \times$ for F. peregrinus and $113.51 \times$ for F. cherrug [18]. Protein-coding genes were predicted while using homology and de novo methods and RNA sequencing data was used to process gene structure. As a result of this combined effort, 16,263 genes were predicted for F. peregrinus and 16,204 were predicted for F. cherrug [18].

Genome sequences available for F. peregrinus and F. cherrug were however not chromosome-level assemblies, but in the form of sub-chromosomal sized scaffolds [18]. The purpose of this study is first to report on (and review) our recent published findings $[6,19]$ in generating chromosome level genome assemblies for F. peregrinus and F. cherrug, second to report novel data on the generation of analyzable karyotypes and ideograms of F. peregrinus, F. cherrug, and F. rusticolis, third to generate a novel, low coverage de-novo genome sequence of $F$. rusticolis falcon, and finally to perform comparative genomics between all three species (two of which are nested under the subgenus hierofalco (F. cherrug and F. rusticolis) and chicken (representing the ancestral avian karyotype).

\section{Materials and Methods}

\subsection{Sample Collection and Chromosome Preparation}

Falcon primary fibroblast cell cultures were prepared from skin biopsies. Sampling was reviewed and approved by the Animal Ethic Committee of CVRL (Central Veterinary Research Laboratory, Dubai, UAE) and Ministry of Climate Change and Environment (MOCCAE), UAE. Avian primary fibroblast cell cultures were prepared from avian tissue samples which include trachea, skin and early stage embryos. Falcon primary fibroblast cell cultures were established only from falcon skin samples. Sampling in this study was reviewed and approved by the Animal Ethic Committee of CVRL, and Ministry of Climate Change and Environment (MOCCAE) UAE, according to the Ministerial Decree No. 384 of the year 2008 on the executive by-law of the Federal Law No. 16 of the year 2007 concerning Animal Welfare. Biopsies were collected at the Dubai Falcon Hospital, UAE. Samples were disaggregated and digested in $3 \mathrm{~mL}$ of HBSS and $5 \mathrm{~mL}$ Trypsin EDTA solution (Sigma, Surrey, UK) and were stirred in a magnetic shaker at $37^{\circ} \mathrm{C}$ for 30 to $45 \mathrm{~min}$. Cells were cultured in Alpha MEM (Fisher, Loughborough, UK) supplemented with 10\% Foetal Bovine Serum (Gibco, Cheshire, UK) and $1 \%$ Pen-Strep-L-Glutamine (Sigma, UK). Flasks were incubated at $40{ }^{\circ} \mathrm{C}$ under $5 \%$. Chromosome suspension preparation followed standard protocols, brief mitostatic treatment with colcemid at a final concentration of $5.0 \mu \mathrm{g} / \mathrm{mL}$ for $1 \mathrm{~h}$ at $40^{\circ} \mathrm{C}$ was followed by hypotonic treatment with $75 \mathrm{mM} \mathrm{KCl}$ for 15 min at $37^{\circ} \mathrm{C}$, and fixation with 3:1 methanol/acetic acid.

\subsection{Fluorescence In-Situ Hybridisation (FISH)}

BAC (Bacterial Artificial Chromosome) selection for cross-species FISH was performed according to Damas et al. [6]. Metaphase preparations were fixed to slides and they were dehydrated through an ethanol series ( 2 min each in $2 \times$ SSC, $70 \%, 85 \%$, and $100 \%$ ethanol at room temperature). FISH probes were mixed in a formamide buffer (Cytocell, Cambridge, UK) with Chicken Hybloc (Insight Biotech, Wembley, UK) and they were applied to the metaphase preparations on a $37^{\circ} \mathrm{C}$ hotplate before sealing with rubber cement prior to simultaneous denaturation on a $75{ }^{\circ} \mathrm{C}$ hotplate. Probe and target DNA were then left to hybridize in a humidified chamber at $37^{\circ} \mathrm{C}$ for $72 \mathrm{~h}$. Slides were washed for $30 \mathrm{~s}$ in $2 \times$ SSC with $0.05 \%$ Tween 20 at room temperature post-hybridization, then counterstained using VECTASHIELD anti-fade medium with DAPI (Vector Labs, Burlingame, CA, USA). Images 
were captured using an Olympus BX61 epifluorescence microscope with cooled CCD camera and SmartCapture 3 (Digital Scientific, Cambridge, UK) system.

\subsection{Genome Mapping}

Recently published Predicted chromosome fragments (PCFs) for the F. cherrug and the F. peregrinus generated using RACA (Reference Assisted Chromosome Assembly) [1] built utilizing the original genome sequences that were generated by Zhan et al. [18] were obtained from O'Connor and co-workers [19]. The zebra finch chromosome level genome assembly and the chicken genome assembly were used as closely related references. For the peregrine falcon, RACA generated 113 PCFs with an N50 of $27.44 \mathrm{Mb}, 57$ of which were placed on chromosomes. For the F. cherrug, RACA generated 103 PCFs with an N50 of $22.27 \mathrm{Mb}$, of which, 64 were placed on chromosomes [19].

Again, as recently published [6,19], a total of 92 BAC clones representing 24 chicken chromosomes were selected from the PCFs bioinformatically and mapped by FISH to the three falcon genomes. Fifty metaphase images were captured for each avian species to create a standard ideogram using Powerpoint. PCFs were ordered on the chromosomes by mapping BAC clones that were associated with PCF directly onto the chromosomes, identifying the chromosomes from the karyotypic data and establishing the order by visual inspection [19].

\subsection{Gyr Falcon Genome Sequencing}

Sequencing of the $F$. rusticolis genome was performed using an Illumina next generation sequencing platform on $300 \mathrm{~kb}$ and $500 \mathrm{~kb}$ libraries. The sequencing and assembly and data analysis process was performed as follows: DNA extracted from 10 birds was pooled and three sequencing libraries prepared, two with fragment sizes of 300 base-pairs (bp) and one with a fragment size of $500 \mathrm{bp}$. These were sequenced on an Illumina Genome Analyser IIx instrument in three lanes generating $150 \mathrm{bp}$ paired-end reads for each library. Additionally, DNA from a single bird was sequenced on an Illumina HiSeq 2000 instrument (500 bp fragment generating 100 bp paired-end reads). Known Illumina primer and adapter sequences were removed and the data trimmed to a $Q$ value of 30. All the sequencing libraries were used as input to SOAPdenovo [20]. The final scaffold $\mathrm{N} 50$ was 32,831, with an assembly length of $1.17 \mathrm{~Gb}$. The data for the gyr falcon assembly 0.2 can be found under BioProject ID (PRJEB27770) and the DOI (http:/ / dx.doi.org/10.7488/ds/2379).

\section{Results}

Here we report the first example of near fully analyzable metaphases of three falcon species (F. peregrinus, F. cherrug and F. rusticolis) with diploid numbers of $2 \mathrm{n}=50$ for F. peregrinus and $2 \mathrm{n}=52$ for F. cherrug and F. rusticolis (Figures 1-3). After some experimentation, a combination of DAPI and propidium iodide gave the sharpest and most distinct banding. Using simple measurement and visual inspection we generated standard ideograms (Figures 1-3). In addition, the smallest chromosome using DAPI staining was disproportionally bright on propidium iodide, and it was thus named chromosome 24 in F. peregrinus and 25 in F. cherrug and F. rusticolis.

For F. rusticolis and F. cherrug: Chromosome 1 has a large pale band near the base, there is a similar but smaller pale band for chromosome 2, and chromosome 4 is easy to distinguish because of its pale band in the centre. Chromosomes 10-16 mostly have two dark bands top and bottom (not dissimilar from a human 14 or 18) and they could be distinguished from one another (looking at subtle differences) with some degree of confidence. Chromosomes 17-25 are generally indistinguishable microchromosomes with the exception of chromosome 25, which is much brighter under propidium iodide. Indeed, chromosome 25 was only visible and distinguishable using propidium iodide, similarly the bright portion of the p-arm of chromosome 12 was much brighter. F. peregrinus is similar, with the two fused chromosomes making up chromosome 1 and the inversions taken into account. 


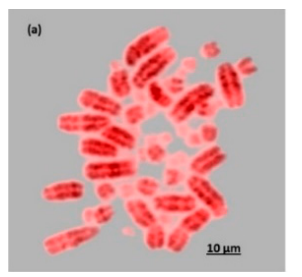

(c)
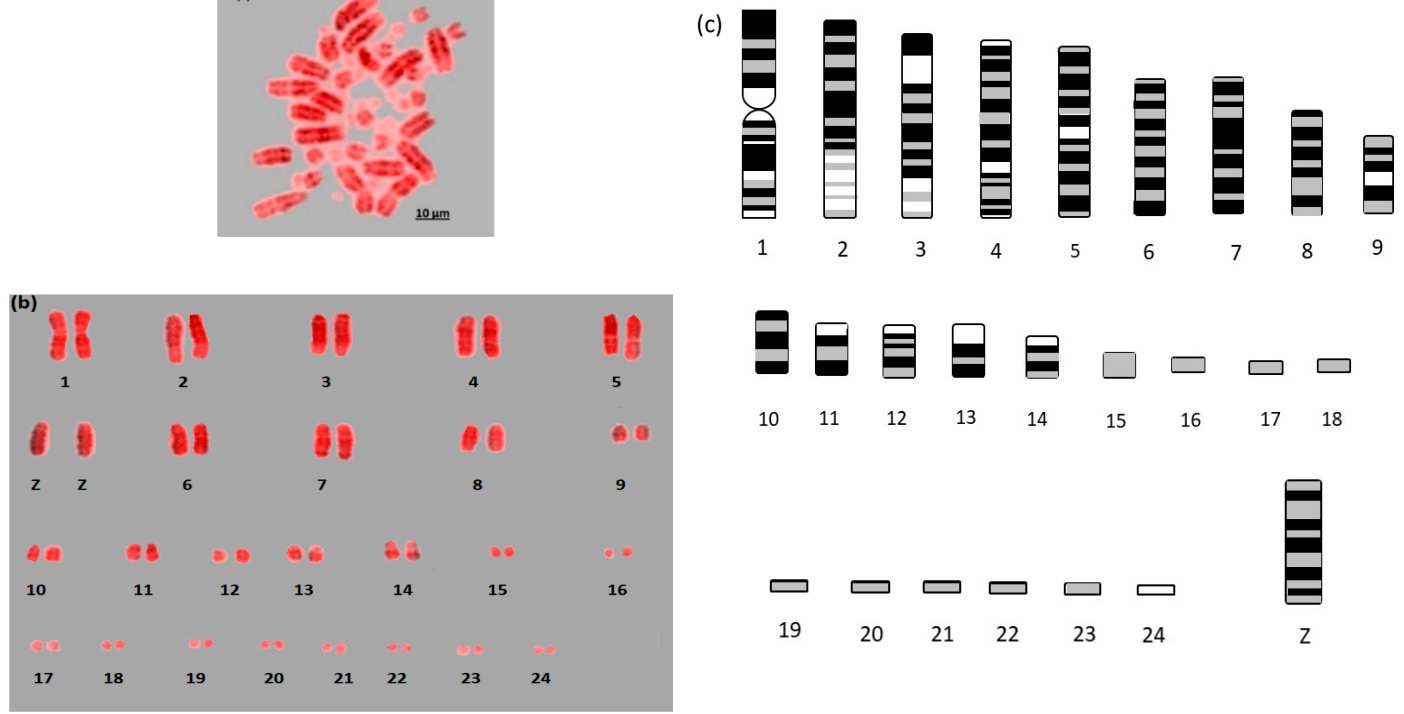

Figure 1. Metaphase preparation (a), karyotype (b), and ideogram (c) of F. peregrinus $(2 \mathrm{n}=50)$.
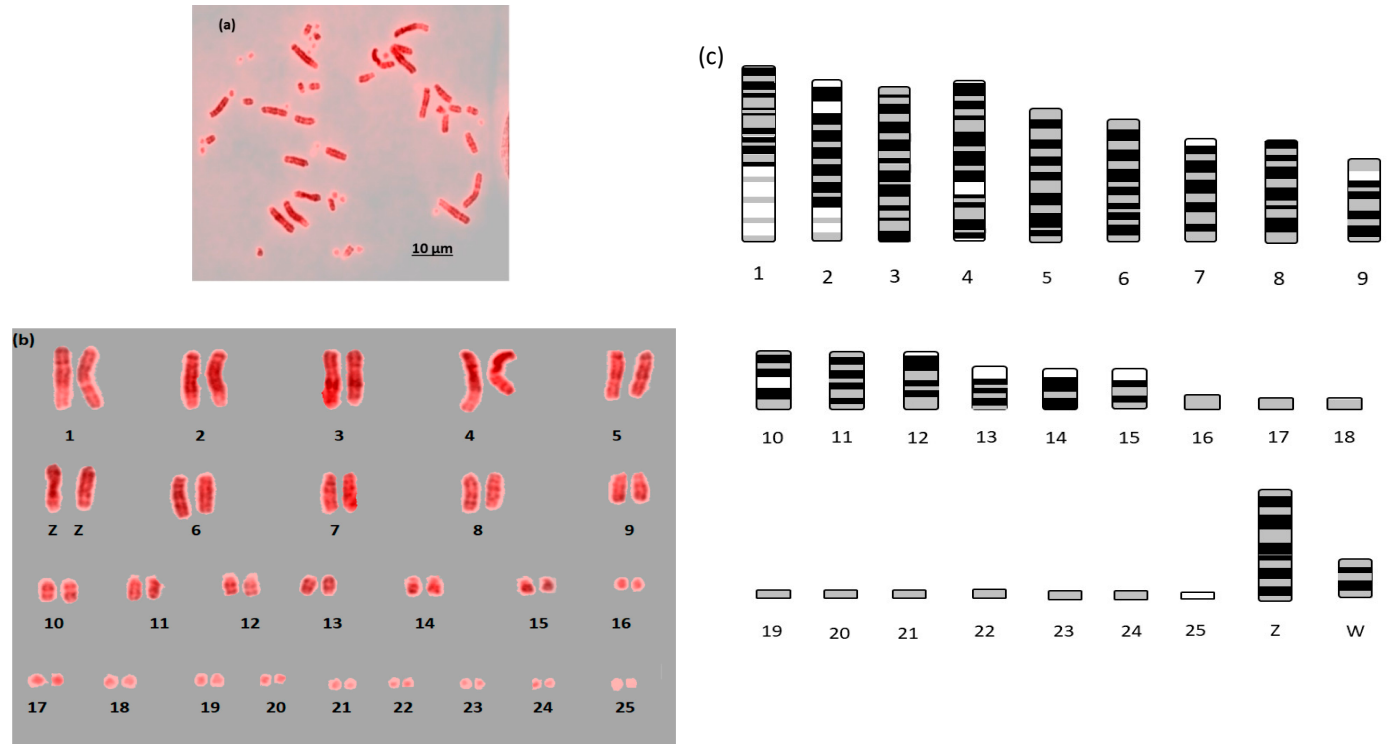

Figure 2. Metaphase preparation (a), karyotype (b), and ideogram (c) of F. cherrug $(2 \mathrm{n}=52)$.

We recently reported the first falcon chromosome level genome assembly [6] through the development of a new approach to upgrade the scaffold-based F. peregrinus genome to chromosome level. This was achieved by using RACA to generate PCFs, combined with the verification of scaffolds by PCR and the physical mapping to chromosome by hybridizing with a universal set of chicken BAC probes by FISH (Figure 4). The Damas et al. [6] study successfully generated a cytogenetically anchored genome map of the F. peregrinus, and it was subsequently repeated for the F. cherrug in 2018 by O'Connor et al. [19]. 

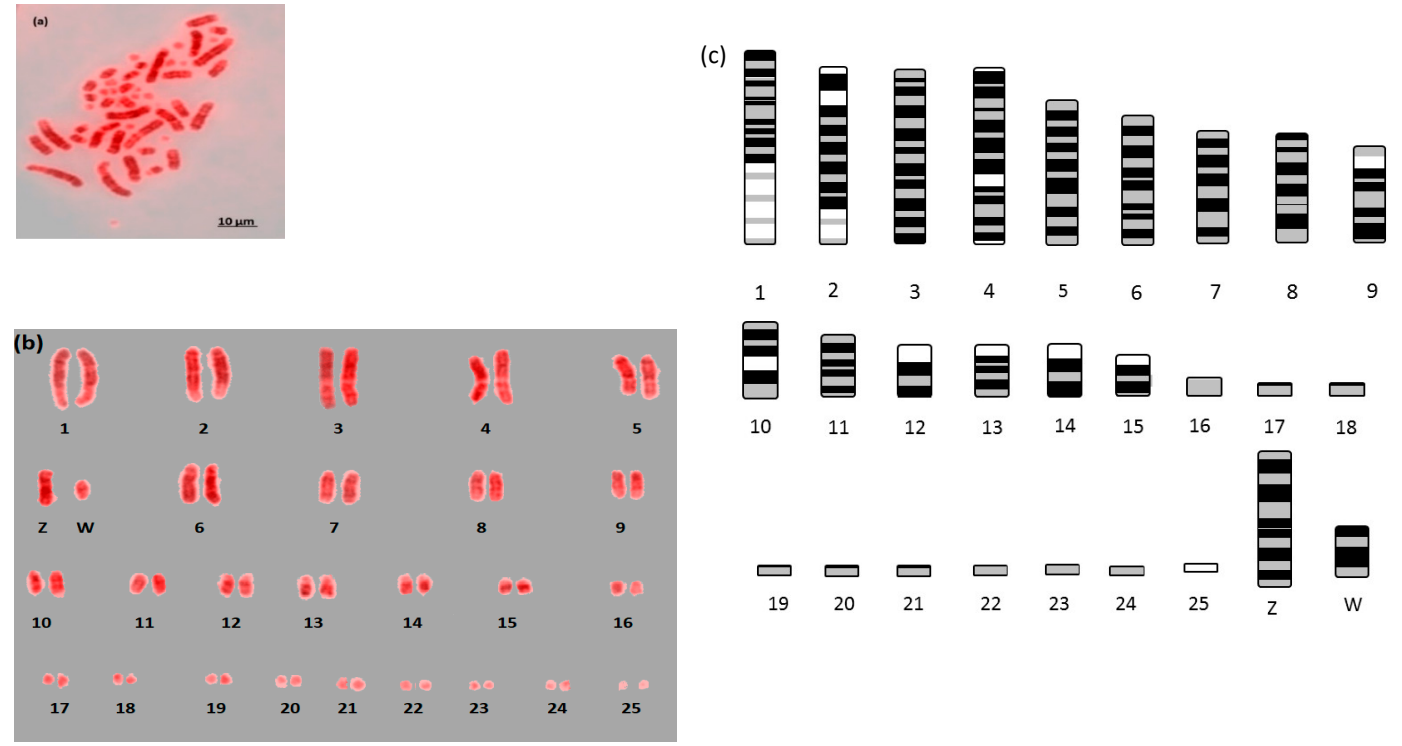

Figure 3. Metaphase preparation (a), karyotype (b), and ideogram (c) of F. rusticolis, $(2 \mathrm{n}=52)$. This is an order of magnitude improvement on the previously published karyotype [10].

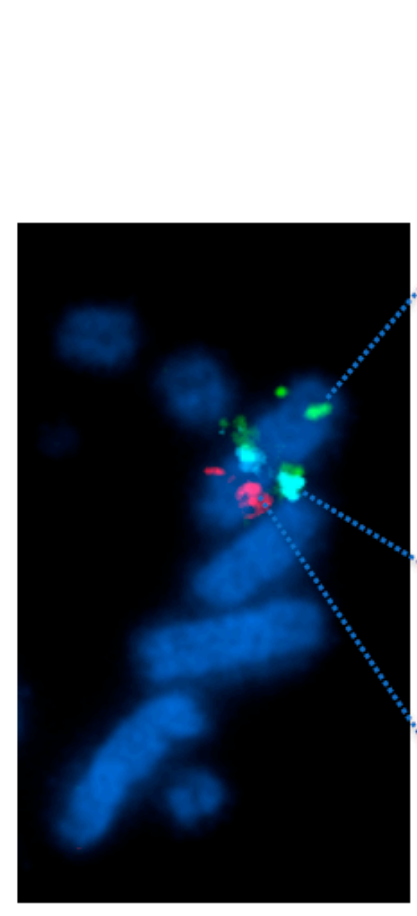

(a)

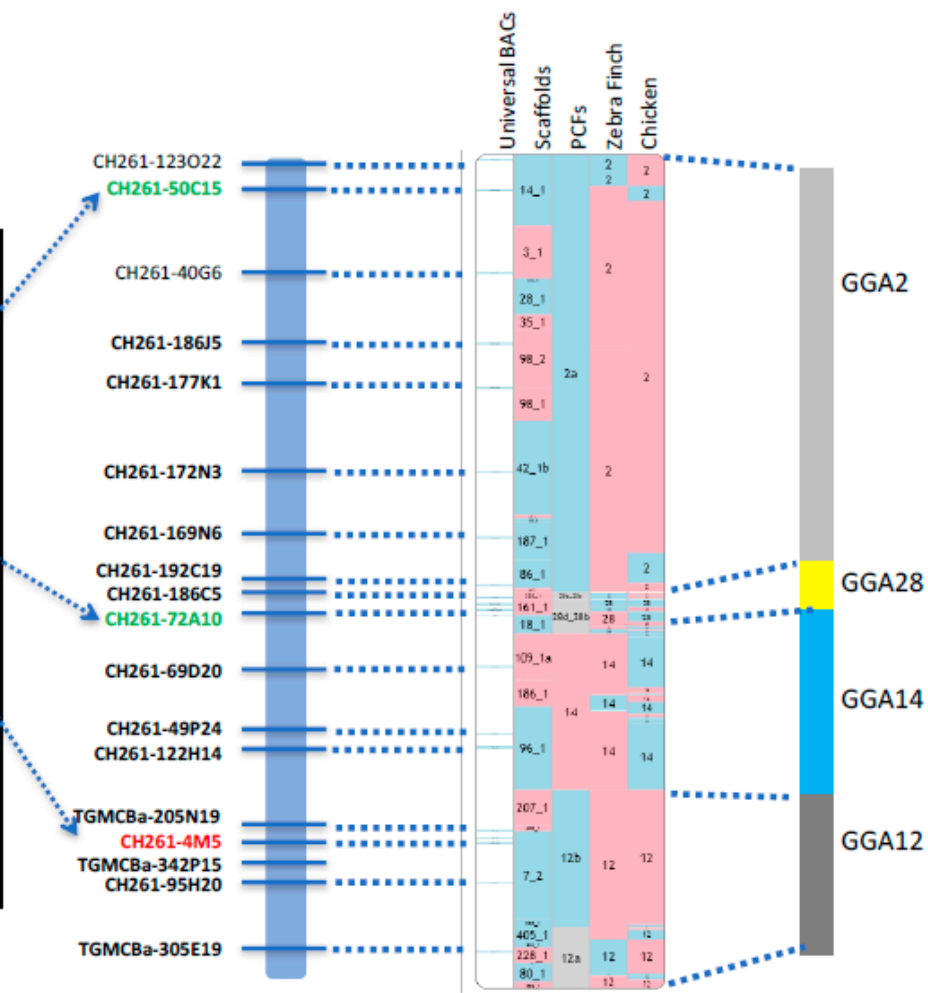

(b)

(c)

(d)

Figure 4. Mapping of scaffolds for F. peregrinus 5 by fluorescence in-situ hybridization (FISH) to create a chromosome level assembly. (a) representative FISH image; (b) position of BACs (Bacterial Artificial chromosomes); (c) Evolution Highway view; and (d) comparative genomics with chicken (GGA).

For the first time we carried out an extensive homology study between the three falcon species (F. peregrinus, F. cherrug and F. rusticolis) and chicken (Figures 5 and 6). FISH was performed with a selected set of BAC clones developed by Damas et al. [6] and identified 13 F. peregrinus specific fusions and five fissions when compared to chicken. F. peregrinus has undergone approximately 38 intrachromosomal rearrangements during the evolution of avian lineages. Comparing homology 
between the chicken and F. cherrug showed that in total, 12 fusions, five fissions, and 36 inversions occurred during evolution from their common ancestor. Moreover, out of 17 mapped chicken microchromosomes, 12 were found to be fused with other chromosomes in both species.
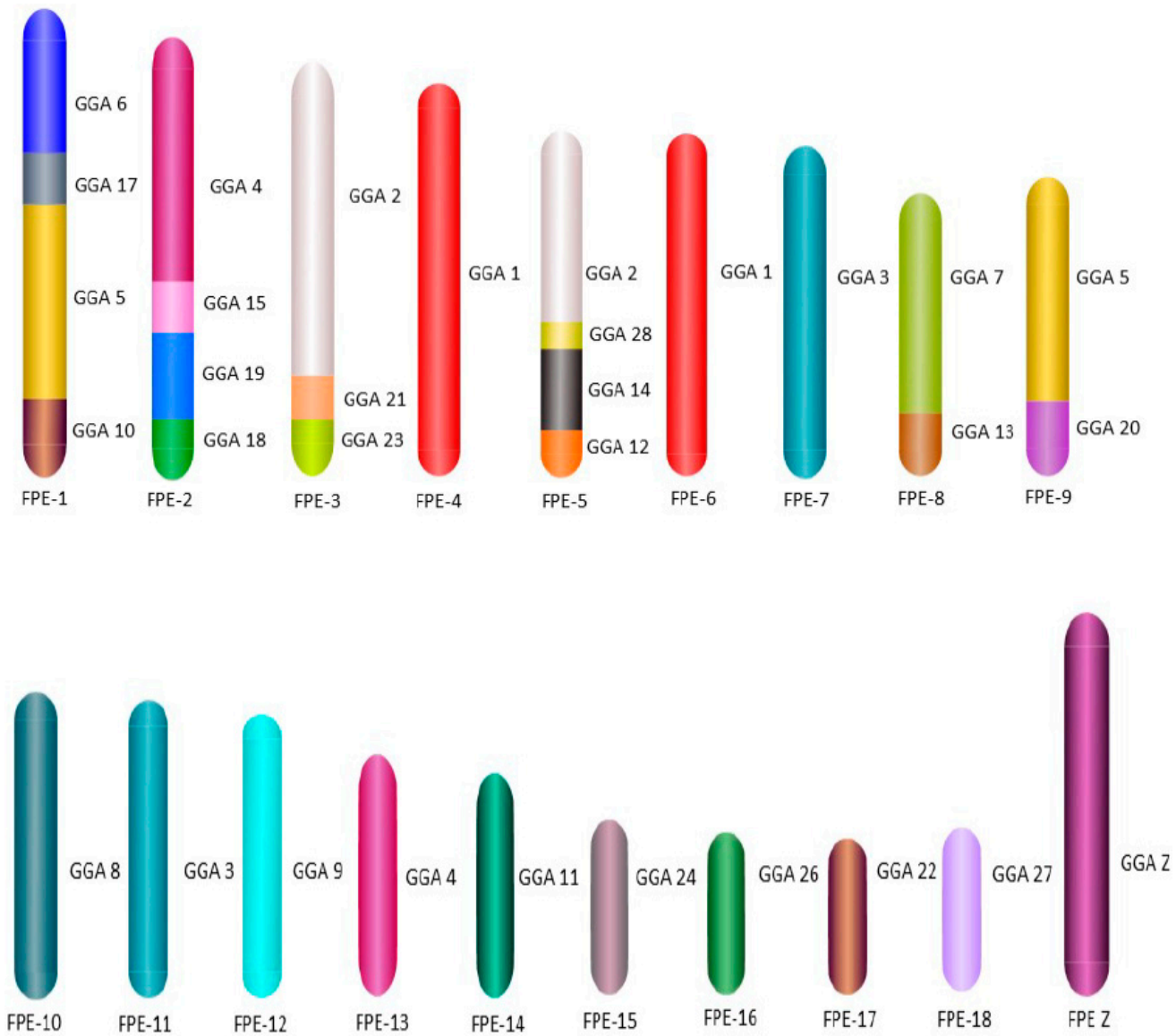

Figure 5. Ideogram representation of F. peregrinus chromosomes and their chicken (GGA) homologs.
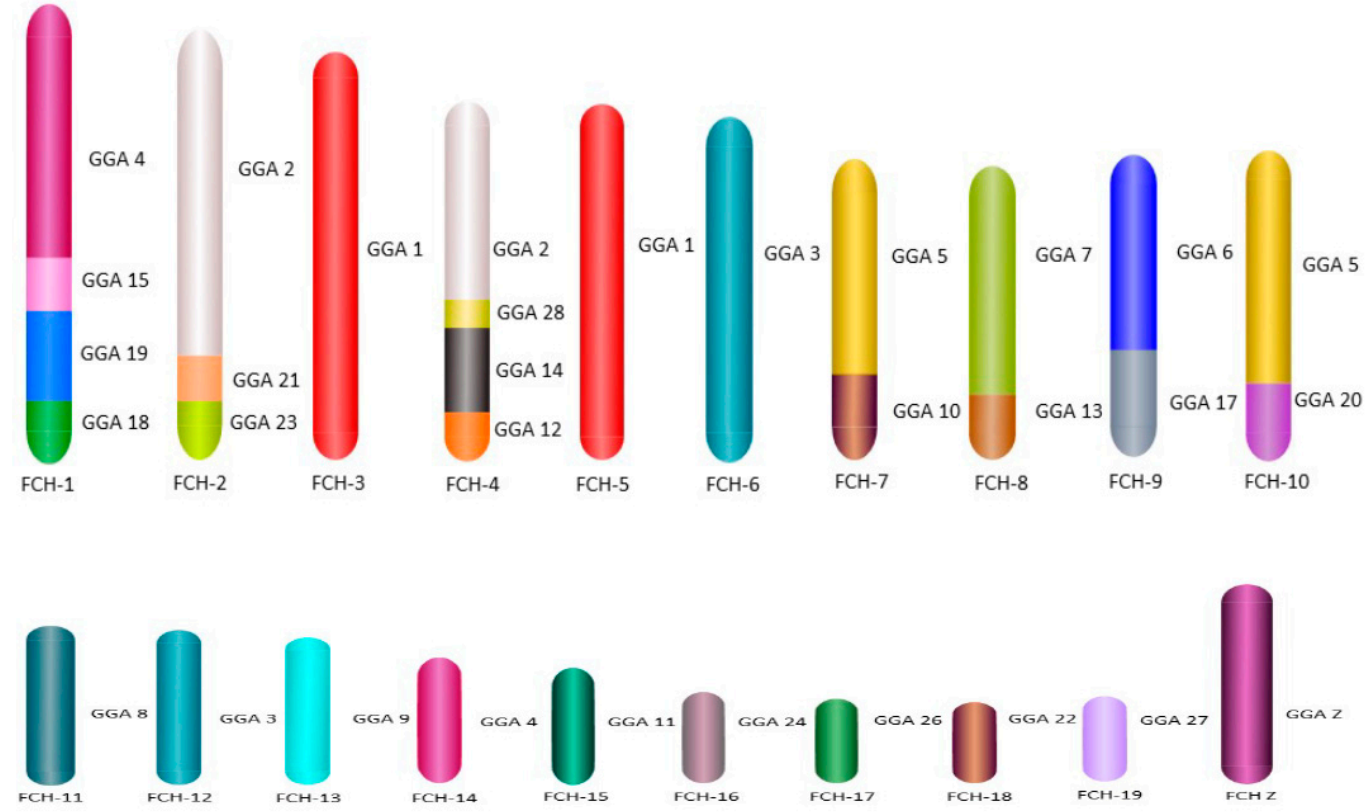

Figure 6. Ideogram representation of F. cherrug and F. rusticolus chromosomes and their chicken (GGA) homologs. 
Comparative mapping of BAC clones between the three species displayed no inter nor intrachromosomal rearrangements between F. rusticolis and F. cherrug. A total of nine intrachromosomal and one interchromosomal changes were identified between F. peregrinus and the two other species (Figure 7 and Figure S1). Finally, we report here, for the first time, the genome sequencing of F. rusticolus (Supplementary Materials).
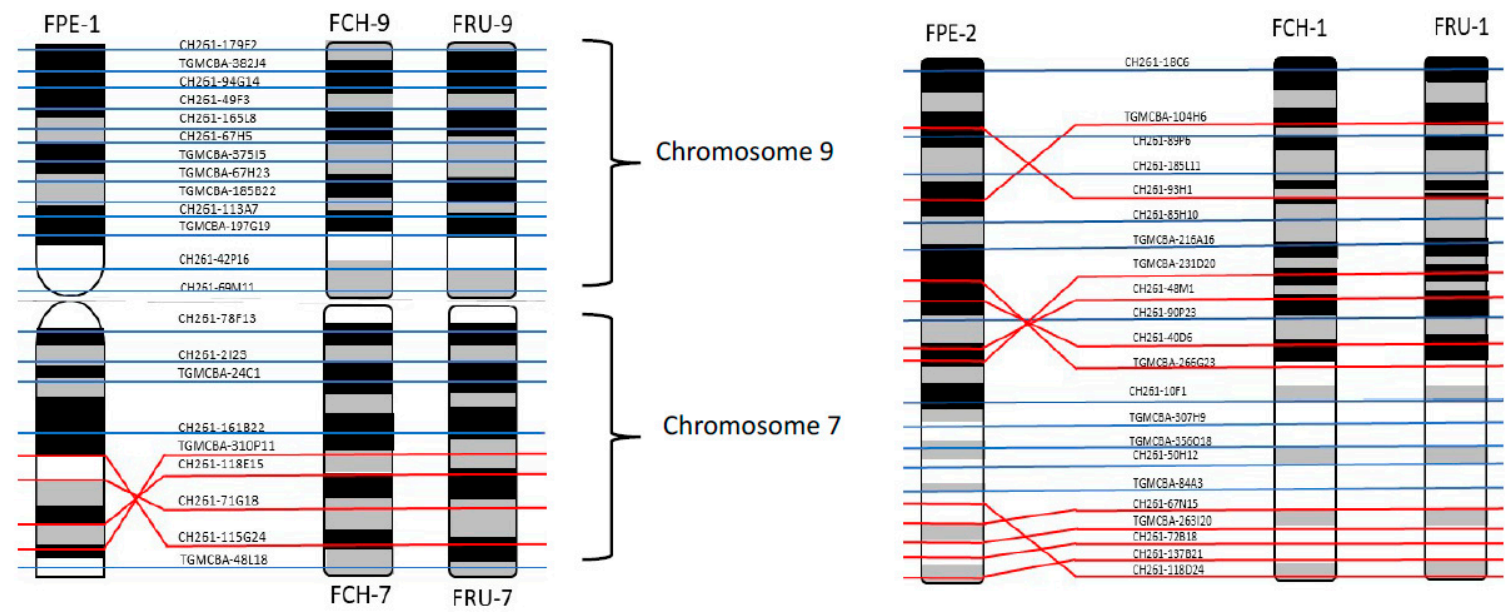

Figure 7. Comparative genomics of F. peregrinus (FPE) chromosomes 1 and 2 and its F. cherrug (FCH) and F. rusticolus (FRU) homologs (with the position of all BACs) revealing one interchromosomal and four intrachromosomal differences. Remaining chromosomes are included in Figure S1.

\section{Discussion}

This study significantly contributed to the understanding of genome organization and evolution in the genus Falco. Specifically, it provides the first example of standard ideograms, analyzable metaphases, chromosome level genome assemblies, and comparative genomics between three species. Our approach combines classical cytogenetics, molecular cytogenetics, and computational algorithms to merge scaffolds into chromosomal fragments. To our knowledge is the only example of genome sequencing in F. rusticolus, and, although a chromosome level assembly was not generated, the evidence shows no difference between the genome organization of F. rusticolus and F. cherrug.

Only partial karyotypes were generated until now for species of the genus Falco. Reasons for a significant improvement in banding patterns are possibly due to the combination of DAPI (which preferentially recognizes AT rich regions of the genome) and propidium iodide (preferentially intercalating between bases), which produced a banding pattern that made karyotyping much clearer. Interestingly, although we have not done an exhaustive study, such a defined banding pattern is not something that we have seen in the chromosomes of other avian species. Whether this is a technical issue borne of the fact that our falcon preparations are fresher than most, or a function of a greater differentiation of AT and GC rich regions in falcons remains to be established.

There are few reports of comparative molecular cytogenetic studies for falcons and only one prior comprehensive comparative FISH study [17]. That study was limited however in that it compared only the largest chromosomes (using chicken macrochromosome paints). It nonetheless provided a baseline for our current data set. The molecular karyotype that is generated in this study and by Damas et al. [6] and O'Connor et al. [19] largely correlates to the preliminary homology study results between chicken and peregrine falcon [17], but it fills in more of the gaps, in particular for the microchromosomes. The chromosome painting data (not shown) and the BAC data generated in these studies suggests a large degree of similarity in the overall genome organization of $F$. cherrug and F. rusticolus when compared with F. tinnunculus. Nishida et al. [17] suggested that $F$. tinnunculus had the ancestral falcon karyotype, and, if this is the case, the same would therefore apply to F. cherrug and F. rusticolus falcons. The lower diploid number of $2 \mathrm{n}=50$ found in the F. peregrinus therefore probably originated from the 
centric fusion of F. cherrug/F. rusticolus chromosomes 7 and 9, forming the metacentric chromosome 1 of the peregrine.

With the genome sequencing of the F. peregrinus and F. cherrug, interest in falcon biology has gained tremendous momentum [18]. Here, we summarize and review our recent efforts to upgrade these scaffold-based genome assemblies to those of the chromosomally assembled genomes for F. peregrinus and F. cherrug, with F. rusticolus implied by its similarity with F. cherrug. The overall strategy used for scaffold assembly by RACA, and physical mapping using a panel of universal BACs [6,20], provides proof of principle for an approach that could be applied to any animal genome. Furthermore, by uploading the chromosomally-assembled genomes to Evolution Highway (F. cherrug and F. peregrinus), users will be able to compare multiple species, with falcons, in order to identify evolutionary breakpoint regions and homologous synteny blocks.

One of the primary benefits of whole genome sequences (particularly chromosome-level assemblies) is to provide a better understanding of evolutionary history of genome organization and chromosome structural variation that is caused by chromosome rearrangements [1]. Multiple projects, including the Bird 10K programme [21], are working to generate draft genome sequences of thousands of extant bird species over the next ten years using next generation sequencing (NGS) technologies to produce de novo assemblies, some of which will be to chromosome-level. Chromosome-level assemblies are also essential for agricultural species where an established order of DNA markers is required to establish phenotype-to-genotype associations for gene-assisted selection and breeding [5]. With this information, high-resolution SNP genotyping is very effective for association studies among different species, which in turn facilitated the mapping of Mendelian disorders, accurate identification of (e.g., cryptic) chromosome translocations [22], discovery of quantitative trait nucleotides (QTNs) and expression quantitative trait loci (eQTLs), and studies of long-range regulatory interactions [23]. This has resulted in significant economic improvement, more efficient food production, and improved global food security in farm animals [23]. The same principles could be applied to establish genomic selection and genome-assisted breeding and/or conservation regimes for falcons.

With these assemblies, comparative genomics also becomes possible in silico [24], particularly when such assemblies are available for multiple species. The comparative genomic maps that are generated here demonstrate the similarities between the three species, with complete synteny between F. cherrug and F. rusticolus. Lack of apparent chromosome rearrangements between these two species raises the question of whether they could be considered the same species. Helbig et al. [25] conducted a phylogenetic relationship study among Falcon species based on cytochrome-b gene variations reporting that the F. cherrug mtDNA haplotypes are almost identical to those of F. rusticolus. Further studies by Nittinger and colleagues used control region and microsatellite markers to elucidate the evolutionary patterns within the hierofalco complex [26,27]. Moreover, F. cherrug and F. rusticolus falcons can produce fertile hybrids in the wild as well as in captivity with extended viability over indefinite generations [28,29]. The detection of hybrid falcons is becoming increasingly important in falcon racing. The nine intrachromosomal differences that were identified between F. peregrinus and F. cherrug/ F. rusticolus could theoretically form the basis for establishing a testing device (FISH based) that could detect hybrids. Such a device could have eight spatially separated hybridization chambers, each of which carries specific DNA FISH probes that are labelled and designed to identify F. peregrinus and F. cherrug/F. rusticolus chromosomes (one for the fusion and seven for the inversions). In recent studies, we have performed multiple (up to 24) hybridizations on single slides for chromosome translocation screening in domestic animals [22], suggesting that a falcon hybrid detection device would be possible to manufacture. Developing such a testing tool to identify F. rusticolus $\times$ F. cherrug falcon hybrids would not be possible as a result of there being no apparent intrachromosomal differences between them.

Falconidae and Accipitridae, together with Psittaciformes members, are recognized as avian species with 'atypical' karyotypes and previous studies have shown that these avian species have the highest numbers of rearrangements occurring on their macrochromosomes [17,30-32]. Recent studies conducted on such 'atypical' karyotype species collectively highlight the substantial amount of 
rearrangements of macrochromosomes that have occurred throughout their evolutionary history. Until now, the only information available was that of their homology to chicken chromosomes GGA 1-9 and Z, while knowledge of their microchromosomal rearrangements has been limited until this and our other recent studies $[6,20]$. By performing microchromosome analysis and thus evaluating the inter-and intrachromosomal relationship between more falcon species, Gyps species and Psittaciformes our understanding of avian chromosome evolution will improve. Moreover, further analysis of the recent availability of several sequenced bird genomes, such as the White-tailed eagle (Accipitriformes), Kea (Psittaciformes), and Turkey vulture (Cathartiformes) [33], provide additional opportunities for insight into the role of evolutionarily conserved synteny blocks in avian evolution. Romanov et al. [34] suggested that chicken was most similar to the common ancestor when compared to ostrich, turkey, duck, budgerigar, and zebra finch. That is, the chicken lineage underwent the fewest number of chromosomal rearrangements. The comparison therefore of falcon interchromosomal and intrachromosomal rearrangements against chicken in this study gives an indication of the number of changes that have occurred in the lineage of the falcons.

In conclusion, the results that are presented here represent the most comprehensive account of chromosomally related genome mapping in falcon species to date. The ideograms presented in the text, the fact that the comparative genomic information is presented on an interactive browser (http:/ / eh-demo.ncsa.uiuc.edu/birds/\#/SynBlocks) and the freely available F. rusticolus data provide a useful resource for the scientific community.

Supplementary Materials: The following are available online at http:/ / www.mdpi.com/1424-2818/10/4/113/s1, Figure S1. Comparative map of the Peregrine falcon (FPE), Saker falcon (FCH) and Gyrfalcon (FRU) chromosomes with the position of all BACs. The red lines indicate inverted segments.

Author Contributions: Conceptualization, D.K.G., R.E.O. and D.M.L.; methodology, S.J., R.E.O., A.F.A.M. and M.W.; software, M.W., and D.M.L; validation, R.E.O., M.W. and D.K.G., formal analysis, S.J., R.E.O., A.F.A.M. and M.W.; investigation, S.J., R.E.O., A.F.A.M. and M.W.; resources, M.W. and D.M.L.; data curation, D.K.G., R.E.O. and M.W.; writing—original draft preparation, S.J., A.F.A.M. and D.K.G..; writing—review and editing, D.K.G. and R.E.O.; visualization, S.J., R.E.O., A.F.A.M., D.M.L. and D.K.G.; supervision, D.K.G.; project administration, D.K.G.; funding acquisition, D.K.G., A.F.A.M. and D.M.L.

Funding: This research was funded by the Biotechnology and Biological Sciences Research Council [BB/K008226/1 and BB/J010170/1 to D.M.L., BB/K008161/1 to D.K.G., and BB/P013732/1 to M.W.]. S.J. was funded by the Government of Dubai via the central Veterinary Research Laboratory.

Acknowledgments: The authors would like to thank the Central Veterinary Research Laboratory, particularly Ali Ridha and Ullrich Wernery for sponsoring S.J. and to Wernery for his scientific input to the project. We would also like to thank Marta Farre-Belmonte (University of Kent, formerly RVC) for her efforts, and that of her students, in assembling (previously published) peregrine and saker falcon genomes.

Conflicts of Interest: The authors declare no conflict of interest. The funders had no role in the design of the study; in the collection, analyses, or interpretation of data; in the writing of the manuscript, or in the decision to publish the results.

\section{References}

1. Kim, J.; Larkin, D.M.; Cai, Q.; Zhang, Y.; Ge, R.L.; Ma, J. Reference-assisted chromosome assembly. Proc. Natl. Acad. Sci. USA 2013, 110, 1785-1790. [CrossRef] [PubMed]

2. França, L.T.C.; Carrilho, E.; Kist, T.B.L. A review of DNA sequencing techniques. Q. Rev. Biophys. 2002, 35, 169-200. [CrossRef] [PubMed]

3. Masabanda, J.S.; Burt, D.W.; O’Brien, P.C.M.; Vignal, A.; Fillon, V.; Walsh, P.S.; Habermann, F. Molecular cytogenetic definition of the chicken genome: Te first complete avian karyotype. Genetics 2004, 166, 1367-1373. [CrossRef] [PubMed]

4. Lewin, H.A.; Larkin, D.M.; Pontius, J.; O’Brien, S.J. Every genome sequence needs a good map. Genome Res. 2009, 19, 1925-1928. [CrossRef] [PubMed]

5. Andersson, L.; Georges, M. Domestic-animal genomics: Deciphering the genetics of complex traits. Nat. Rev. Genet. 2004, 5, 202-212. [CrossRef] [PubMed] 
6. Damas, J.; O'Connor, R.; Farré, M.; Lenis, V.P.E.; Martell, H.J.; Mandawala, A.; Larkin, D.M. Upgrading short-read animal genome assemblies to chromosome level using comparative genomics and a universal probe set. Genome Res. 2017, 27, 875-884. [CrossRef] [PubMed]

7. Griffin, D.K.; Robertson, L.B.W.; Tempest, H.G.; Skinner, B.M. The evolution of the avian genome as revealed by comparative molecular cytogenetics. Cytogenet. Genome Res. 2007, 117, 64-77. [CrossRef] [PubMed]

8. De Boer, L.E.M. Karyological heterogeneity in the Falconiformes (aves). Experientia 1975, 31, 1138-1139. [CrossRef] [PubMed]

9. Schmutz, S.M.; Oliphant, L.W. Chromosome study of peregrine, prairie, and gyrfalcons with implications for hybrids. J. Hered. 1987, 78, 388-390. [CrossRef]

10. Amaral, K.; Felipe, K.; Jorge, W. The chromosomes of the Order Falconiformes: A review. Ararajuba 2003, 11, 65-73.

11. Sibley, C.G.; Charles, G.; Ahlquist, J.E. Phylogeny and Classification of Birds: A study in Molecular Evolution; Yale University Press: New Haven, CT, USA, 1990.

12. Del Hoyo, J.; Elliott, A.; Christie, D. Cotingas to Pipits and Wagtails. In Handbook of the Birds of the World; Oxford University Press: Oxford, UK, 1992.

13. Itoh, Y.; Arnold, A.P. Chromosomal polymorphism and comparative painting analysis in the zebra finch. Chromosome Res. 2005, 13, 47-56. [CrossRef] [PubMed]

14. Belterman, R.H.R.; De Boer, L.E.M. A karyological study of 55 species of birds, including karyotypes of 39 species new to cytology. Genetica 1984, 65, 39-82. [CrossRef]

15. De Boer, L.E.M. The somatic chromosome complements of 16 species of falconiformes (Aves) and the karyological relationships of the order. Genetica 1990, 46, 77-113. [CrossRef]

16. Longmire, J.L.; Lewis, A.K.; Brown, N.C.; Buckingham, J.M.; Clark, L.M.; Jones, M.D.; Ray, F.A. Isolation and molecular characterization of a highly polymorphic centromeric tandem repeat in the family Falconidae. Genomics 1988, 2, 14-24. [CrossRef]

17. Nishida, C.; Ishijima, J.; Kosaka, A.; Tanabe, H.; Habermann, F.A.; Griffin, D.K.; Matsuda, Y. Characterization of chromosome structures of Falconinae (Falconidae, Falconiformes, Aves) by chromosome painting and delineation of chromosome rearrangements during their differentiation. Chromosome Res. 2008, 16, 171-181. [CrossRef] [PubMed]

18. Zhan, X.; Pan, S.; Wang, J.; Dixon, A.; He, J.; Muller, M.G.; Bruford, M.W. Peregrine and saker falcon genome sequences provide insights into evolution of a predatory lifestyle. Nat. Genet. 2013, 45, 563-566. [CrossRef] [PubMed]

19. O'Connor, R.E.; Farre, M.; Joseph, S.; Damas, J.; Kiazim, L.G.; Jennings, R.; Griffin, D.K. Chromosome-level assembly reveals extensive rearrangement in saker falcon, budgerigar but not ostrich genomes. Genome Biol. 2018, in press.

20. Li, R.; Zhu, H.; Ruan, J.; Qian, W.; Fang, X.; Shi, Z.; Wang, J. De novo assembly of human genomes with massively parallel short read sequencing. Genome Res. 2010, 20, 265-272. [CrossRef] [PubMed]

21. Zhang, G.; Rahbek, C.; Graves, G.R.; Lei, F.; Jarvis, E.D.; Gilbert, M.T.P. Genomics: Bird sequencing project takes off. Nature 2015, 522, 34. [CrossRef] [PubMed]

22. O'Connor, R.E.E.; Fonseka, G.; Frodsham, R.; Archibald, A.L.L.; Lawrie, M.; Walling, G.A.A.; Griffin, D.K.K. Isolation of subtelomeric sequences of porcine chromosomes for translocation screening reveals errors in the pig genome assembly. Anim. Genet. 2017, 48, 395-403. [CrossRef] [PubMed]

23. Hu, X.; Gao, Y.; Feng, C.; Liu, Q.; Wang, X.; Du, Z.; Li, N. Advanced technologies for genomic analysis in farm animals and its application for QTL mapping. Genetica 2009, 136, 371-386. [CrossRef] [PubMed]

24. Farré, M.; Narayan, J.; Slavov, G.T.; Damas, J.; Auvil, L.; Li, C.; Larkin, D.M. Novel Insights into Chromosome Evolution in Birds, Archosaurs, and Reptiles. Genome Biol. Evol. 2016, 8, 2442-2451. [CrossRef] [PubMed]

25. Helbig, A.J.; Seibold, I.; Bednarek, W.; Brüning, H.; Gaucher, P.; Ristow, D.; Wink, M. Phylogenetic relation-ships among Falcon species (genus Falco) according to DNA sequence variation of the cytochrome b gene. Raptor Conserv. Today 1994, 593-599.

26. Nittinger, F.; Haring, E.; Pinsker, W.; Wink, M.; Gamauf, A. Out of Africa? Phylogenetic relationships between Falco biarmicus and the other hierofalcons (Aves: Falconidae). J. Zool. Syst. Evol. Res. 2005, 43, 321-331. [CrossRef] 
27. Nittinger, F.; Gamauf, A.; Pinsker, W.; Wink, M.; Haring, E. Phylogeography and population structure of the saker falcon (Falco cherrug) and the influence of hybridization: Mitochondrial and microsatellite data. Mol. Ecol. 2007, 16, 1497-1517. [CrossRef] [PubMed]

28. Eastham, C.P.; Nicholls, M.K. Morphometric Analysis of Large Falco Species and Their Hybrids With Implications for Conservation. J. Raptor Res. 2005, 39, 386-393.

29. Heidenreich, M. Birds of Prey: Medicine and Management; Blackwell Science: Oxford, UK, 1997.

30. de Oliveira Furo, I.; Kretschmer, R.; O’Brien, P.C.; Ferguson-Smith, M.A.; de Oliveira, E.H.C. Chromosomal Diversity and Karyotype Evolution in South American Macaws (Psittaciformes, Psittacidae). PLoS ONE 2015, 10, e0130157. [CrossRef] [PubMed]

31. Nie, W.; O’Brien, P.C.M.; Fu, B.; Wang, J.; Su, W.; He, K.; Baker, R. Multidirectional chromosome painting substantiates the occurrence of extensive genomic reshuffling within Accipitriformes. BMC Evol. Biol. 2015, 15, 205. [CrossRef] [PubMed]

32. Seibold-Torres, C.; Owens, E.; Chowdhary, R.; Ferguson-Smith, M.A.; Tizard, I.; Raudsepp, T. Comparative Cytogenetics of the Congo African Grey Parrot (Psittacus erithacus). Cytogenet. Genome Res. 2016, 147, 144-153. [CrossRef] [PubMed]

33. Zhang, G.; Li, C.; Li, Q.; Li, B.; Larkin, D.M.; Lee, C.; Froman, D.P. Comparative genomics reveals insights into avian genome evolution and adaptation. Science 2014, 346, 1311-1320. [CrossRef] [PubMed]

34. Romanov, M.N.; Farré, M.; Lithgow, P.E.; Fowler, K.E.; Skinner, B.M.; O'Connor, R.; Nishida, C. Reconstruction of gross avian genome structure, organization and evolution suggests that the chicken lineage most closely resembles the dinosaur avian ancestor. BMC Genom. 2014, 15, 1060. [CrossRef] [PubMed]

(C) 2018 by the authors. Licensee MDPI, Basel, Switzerland. This article is an open access article distributed under the terms and conditions of the Creative Commons Attribution (CC BY) license (http:/ / creativecommons.org/licenses/by/4.0/). 